\title{
Commentary
}

\section{Problematic substance use or problematic substance use policies?}

\author{
Tim Stockwell, PhD (1,2); Cecilia Benoit, PhD (1,3); Kiffer Card, PhD (1); Adam Sherk, PhD (1)
}

Tweet this article

\begin{abstract}
This special issue on substance use issues comes at a critical time for Canadian health policy makers and researchers. Most attention is currently focussed on the opioid crisis and the potential impacts of cannabis legalization. However, our most widely used and harmful substances continue to be alcohol and nicotine. Our policies to reduce harms from these substances are failing. While alcohol control policies are being gradually abandoned, opportunities to maximize the harm reduction potential of new, alternative and safer nicotine delivery devices are not being grasped. More generally, a greater focus is needed on harm reduction strategies that are informed by the experience of marginalized people with severe substance use-related problems so as to not exacerbate health inequities.

In order to better inform policy responses, we recommend innovative approaches to monitoring and surveillance that maximize the use of multiple data sources, such as those used in the Canadian Substance Use Costs and Harms (CSUCH) project. Greater attention to precision in defining patterns of risky use and harms is also needed to support policies that more accurately reflect and respond to actual levels of substance userelated harm in Canadian society.
\end{abstract}

Keywords: substance use, alcohol, cannabis, tobacco, smoking, harm reduction, surveillance, surveys, public health, health inequities

\section{Introduction}

Substance use costs the Canadian economy an estimated $\$ 46$ billion per year in direct health care expenditures, lost productivity and expenses related to enforcing its Criminal Code. The great majority of these costs result from alcohol and tobacco use. ${ }^{1}$ The Canadian Drugs and Substances Strategy (CDSS) is the federal government's official response to these rising costs and societal harms. The CDSS focusses on optimizing four activitiesprevention, treatment, harm reduction and enforcement-through increased funding for research and programming. The primary focus of the CDSS, however, is on cannabis, opioids and other illicit substances.
In 2018, Health Canada conducted a broadbased evaluation of the federal government's strategy. This evaluation highlighted many fundamental problems with these four activities. This special issue on problematic substance use in Canada casts further light on the challenges. Aside from conceptualizing problematic substance use for many drugs, legal and illegal, this issue of Health Promotion and Chronic Disease Prevention in Canada also describes programs and policies that are failing to adequately address the costs and harms related to substance use.

A key starting point for this commentary is the principle that priorities for substance use policies in Canada need to
Highlights

- Substance use in Canada cost $\$ 46$ billion in 2017, with the great majority of these costs resulting from the use of tobacco and alcohol.

- Substance use-related costs, harms and rates of use have been increasing in Canada over the past decades for both legal and illegal substances, including those for cannabis

- Canadian policies to address our most harmful substances, i.e. alcohol, cannabis and tobacco, are largely failing, despite significant opportunities to improve polices on pricing, taxation and marketing of legal substances.

- Canada's monitoring and surveillance efforts can be improved by developing more discerning measures of risk and maximizing the use of multiple data sources.

reflect their potential for reducing related harms. Another is that policies are informed by the experiences of marginalized groups so as not to exacerbate health inequities. We provide examples of Canadian policy directions that are failing to address, and are even exacerbating, harms due to alcohol and tobacco use. In addition, broad system-wide policies addressing some of the social determinants of health at all stages in the lifespan need to be prioritized as they may have benefits across the full range of types of substance use and related harms.

This special issue contains eight articles that variously evaluate policies, describe

\section{Author references:}

1. Canadian Institute for Substance Use Research, University of Victoria, Victoria, British Columbia, Canada

2. Department of Psychology, University of Victoria, Victoria, British Columbia, Canada

3. Department of Sociology, University of Victoria, Victoria, British Columbia, Canada

Correspondence: Tim Stockwell, Canadian Institute for Substance Use Research, University of Victoria, PO Box 1700 STN CSC, Victoria, BC V8W 2Y2; Tel: 250-472-5445;

Email: timstock@uvic.ca 
current trends in substance use and examine risk factors. Four articles focus on alcohol policies, three on patterns of harm from cannabis use and one on potential protective factors against substance use among youth. While many important substances and relevant concerns are not captured here (e.g. stigma, marginalized populations, social determinants), these articles present salient and timely perspectives on some of the biggest challenges facing Canada right now. Indeed, with the legalization of cannabis in 2018, there has been a flurry of studies aiming to understand what this means for the prevalence of cannabis (especially among youth) and future patterns of substance use, related harms and possible benefits. This interest in cannabis has further sidelined studies on alcohol and nicotine, despite these continuing to be the greatest contributors to substance use-related costs and harms.

The authors of these papers have also provided a number of recommendations for next steps and future directions to support policy- and decision-makers. These include the need for (1) continued monitoring and surveillance of substance use and substance use-related harms; (2) legislative and regulatory mechanisms to reduce the harms arising from substance use, such as excise taxes and minimum unit pricing for legalized drugs; and (3) enhanced education and programming for youth, families and communities in Canada.

We take the opportunity here to dive a bit deeper into these three broad areas.

\section{Monitoring and surveillance}

Canada has a strong system of monitoring and surveillance in place; however, there are avenues for improvement. Foundational for monitoring substance use and related harms are national substance use surveys, administered by Statistics Canada and Health Canada. These provide a picture of substance use, although delays in data release mean there are difficulties in identifying emerging trends. Programs such as the Canadian Community Epidemiology Network on Drug Use help to fill these gaps in sentinel surveillance by connecting community partners across Canada and focussing on identifying emerging trends and potential problems with safe supply.

The Statistics Canada and Health Canada surveys support broader studies of substance use, such as the Canadian Substance Use Costs and Harms (CSUCH) project, a national study of the economic cost of substance use. CSUCH compares costs and harms related to eight categories. ${ }^{1}$ The overall economic cost of substance use across health care, lost productivity, criminal justice and other direct cost domains was estimated at $\$ 46$ billion in 2017. Accounting for more than threequarters of the total cost are the three legal drugs: alcohol ( $\$ 16.6$ billion), tobacco ( $\$ 12.3$ billion) and cannabis ( $\$ 3.2$ billion). Among illicit substances, only opioids ( $\$ 5.9$ billion) and cocaine ( $\$ 3.7$ billion) make up more than $5 \%$ of the total cost.

Monitoring efforts may be prioritized using two criteria: overall population-level harm, where alcohol and tobacco have by far the largest contribution, and significant emerging health concerns, such as the opioid crisis.

Several articles in this special issue contribute toward providing baseline knowledge of cannabis poisoning and/or injury harms over the years leading up to legalization. Maloney-Hall et $\mathrm{al}^{2}$ found that rates of hospital-based cannabis use disorders more than doubled from 2006 to 2015. Champagne et al. ${ }^{3}$ found increases in cannabis-related poisonings and injuries in the lead up to legalization among both adults and youth, while Cheng et al. ${ }^{4}$ reported that the majority of poisonings related to cannabis occurred alongside alcohol co-use. Continued monitoring of cannabis harms, with the concurrent use of other substances, is clearly a priority post legalization. We recommend this is accompanied with more precise definitions of patterns of cannabis use and related harms, including the quantification of amounts used. Alcohol-related harms are mostly dose-related and it is insufficient to only record use patterns in terms of frequency. ${ }^{5}$ Ignoring the amount of cannabis consumed per occasion could lead to an underestimation of health consequences. $^{6}$

Monitoring of substance use in Canada should continue to be a focus, with improvements in data collection. For example, despite particular concerns, national surveys currently do not collect information on substance use in the territories. Survey questions should collect information on use and harms, and also on emerging harm reduction opportunities, such as substituting nicotine vaping for tobacco smoking or, potentially, cannabis for the use of alcohol and other substances.

\section{Legislative and policy mechanisms}

Three of the papers in this special issue of the Journal highlight specific opportunities for Canadian governments to use policies related to alcohol to improve public health outcomes. Paradis et al. ${ }^{7}$ show that regulations to restrict the content of alcohol promotions in traditional media need to be extended to modern social media. In particular, they show that bars frequented by students in four Canadian cities routinely allow posts on their social media accounts that violate existing regulations. Stockwell et al. ${ }^{8}$ identify alternative alcohol price and tax policies that could save hundreds of Canadian lives and prevent thousands of hospital admissions each year. They also estimate that failures over the past 25 years to adjust alcohol excise taxes for inflation have cost the federal government about $\$ 11$ billion. Sherk ${ }^{9}$ shows that the revenues currently received by federal government fall short by one-third of the estimated national economic costs generated by alcohol.

The Canadian Alcohol Policy Evaluation (CAPE) project ${ }^{10,11}$ paints a broader picture of regulatory failure regarding alcohol and public health on the part of Canadian governments. Applying more than 200 indicators across 11 evidence-based policy domains, the CAPE project gave failing grades to provincial and territorial governments for their implementation of most alcohol policies. While many strong practices were identified, there is a trend for provincial and territorial governments to introduce policies that worsen public health outcomes from alcohol, such as the "buck a beer" program and sales in grocery stores (as described by, e.g. Myran et al., 2019). ${ }^{12}$ Van der Maas et al. ${ }^{13}$ demonstrated the viability and potential value of a Canadian arm of the International Alcohol Control study to monitor and evaluate alcohol policies longitudinally.

Central to a modern public health approach to alcohol policy is the principle that the more alcohol is consumed by a population, the greater the overall risk to that population's health and safety. ${ }^{14}$ With growing evidence for effective policies to reduce population alcohol consumption, it would be timely to apply lessons learned 
from alcohol policy to our other legal drugs. In this issue, three papers focus on trends in and/or levels of cannabis-related harms as tracked in Canadian surveillance systems. Maloney-Hall et al. ${ }^{2}$ show that between 2006 and 2015, rates of cannabisrelated hospitalizations doubled. Almost half of these were identified as "mental and behavioural disorders" including "psychotic disorders," which tripled in number. Champagne et al. ${ }^{3}$ report a 30\% increase in emergency room presentations for cannabis-related poisonings or injuries between 2015 and 2018. Elsewhere, analyses of Canadian substance use self-report surveys conducted for the CSUCH project confirm parallel increases in rates of cannabis use between 2007 and 2017. ${ }^{1}$ These trends highlight the need for more effective cannabis policies such as those identified for alcohol in the CAPE studies in such domains as pricing and taxation, availability and controls on marketing.

\section{Enhanced education and programming for marginalized groups}

Strong population-wide policies need to be tempered in order to help reduce harms to people with severe substance-use problems, many of whom are also marginalized and stigmatized. Managed alcohol programs are a uniquely Canadian harm reduction approach designed to limit harms experienced by unstably housed people, who in many parts of Canada, are overrepresented by Indigenous peoples. They involve the provision of accommodation coupled with regular administration of alcohol in a safe environment. ${ }^{15}$ Work is underway to explore whether substituting cannabis for alcohol further reduces harms for this population. ${ }^{16}$ In relation to nicotine, we need to maximize the harm reduction potential of alternative, safer methods of use in order to further reduce the continuing tragic epidemic of tobacco-related lung diseases. In 2018, the United States National Academies of Science, Medicine and Engineering concluded that e-cigarettes are "far less harmful" than regular tobacco cigarettes. ${ }^{17}$ Smoking dried cannabis leaves, especially in combination with tobacco, also poses serious risks of lung disease. ${ }^{18}$ Policies that restrict access to vaping equipment and supplies for heavy smokers, especially for low-income and marginalized groups, risk losing the tremendous potential benefits from this new technology for reduction of the almost 50000 deaths estimated each year from smoking-related lung disease. ${ }^{1}$

Community-based harm reduction approaches are also recommended for substance-using pregnant and parenting women, who are overrepresented by lowincome Indigenous women. These programs need to be assessed so that providers carefully weigh the interests and concerns of parents and the welfare of their unborn and dependent children. Benoit et al. ${ }^{19}$ found health and social care service providers, even when embracing harm reduction principles, tended to view any substance use by women who were pregnant or had recently become parents as problematic/morally wrong. By contrast, new parents, even when holding abstinence as the ideal, recognized the autonomy of women to judge substance use risk for themselves. Participants also called attention to social structural factors (unstable housing, food insecurity, violence, etc.) that increase harms associated with such substance use. ${ }^{20}$ Harm reduction programs aiming to provide nonjudgmental care for marginalized women and their families need to measure "success" from multiple perspectives in order to assess the quality and impact of care, improve services and apply this learning to future program development. ${ }^{21}$

The study by Enns and Orpana ${ }^{22}$ of factors such as resilience and autonomy that may protect youth from harmful substance use is a timely reminder that prevention needs to focus on strengths and not just vulnerabilities.

\section{Conclusions}

We conclude that our current substance use policies are largely failing or at least failing to reach their potential, especially for our traditional legal drugs. Canada needs to strengthen monitoring and surveillance and augment this with modelling approaches such as those applied in the CSUCH national monitoring project to achieve more comprehensive and accurate estimates of patterns of substance use and related harms. ${ }^{1}$ Underpinning our policy responses should be the principle of prioritizing strategies to deal with those substances that cause the greatest harm while also developing harm reduction strategies for marginalized, heavy-using populations. In monitoring and surveillance activities we should not conflate all substance use with substance use-related harms. We need more precise definitions and measures of the specific patterns of use that pose the greatest harm while acknowledging the right of citizens to access psychoactive substances and participate in the process of implementing harm reductions strategies.

In closing, the editors would like to thank the authors for the exceptional work they have invested in these studies and the editorial team at Health Promotion and Chronic Disease Prevention in Canada for putting together this special issue.

\section{Conflicts of interest}

TS, CB, KC and AS were Guest Editors for this issue of the HPCDP Journal, but removed themselves from the editorial decision-making associated with this manuscript.

\section{Statement}

The content and views expressed in this article are those of the authors and do not necessarily reflect those of the Government of Canada.

\section{References}

1. Canadian Substance Use Costs and Harms Scientific Working Group. Canadian substance use costs and harms (2007-2017). Ottawa (ON): Canadian Centre on Substance Use and Addiction; 2020. Forthcoming.

2. Maloney-Hall B, Wallingford S, Konefal S, Young M. Psychotic disorder and cannabis use: Canadian hospitalization trends, 2006-2015. Health Promot Chronic Dis Prev Can. 2020;40(5/6): 176-83. doi:10.24095/hpcdp.40.5/6.06.

3. Champagne A, McFaull S, Thompson W, Bang F. Surveillance from the high ground: sentinel surveillance of injuries and poisonings associated with cannabis. Health Promot Chronic Dis Prev Can. 2020;40(5/6):184-92. doi: 10.24095/hpcdp.40.5/6.07.

4. Cheng P, Zargaran A, Rajabali F, Turcotte K, Babul S. Setting the baseline: a description of cannabis poisonings at a Canadian pediatric hospital prior to the legalization of recreational cannabis. Health Promot Chronic Dis Prev Can. 2020;40(5/6):193-200. doi:10.24095/hpcdp.hpcdp.40.5/6.08. 
5. Zeisser C, Thompson K, Stockwell T, et al. A "standard joint"? The role of quantity in predicting cannabis-related harm. Addict Res Theory. 2012;20(1): 82-92. doi:10.3109/16066359.2011 .569101 .

6. Asbridge M, Duff C, Marsh DC, Erickson PG. Problems with the identification of 'problematic' cannabis use: examining the issues of frequency, quantity, and drug use environment. Eur Addict Res. 2014;20(5): 254-67. doi:10.1159/000360697.

7. Paradis C, Zhao J, Stockwell T. What popular bars post on social media platforms: a case for improved alcohol advertising regulation. Health Promot Chronic Dis Prev Can. 2020; 40(5/6):143-52. doi:10.24095/hpcdp $.40 .5 / 6.03$.

8. Stockwell T, Churchill S, Sherk A, Sorge J, Gruenewald P. How many alcohol attributable deaths and hospital admissions could be prevented by alternative pricing and taxation policies? Modelling impacts on alcohol consumption, revenues and related harms in Canada. Health Promot Chronic Dis Prev Can. 2020;40(5/6): 153-64. doi:10.24095/hpcdp.40.5/6.04.

9. Sherk A. The alcohol deficit: Canadian government revenue and societal costs from alcohol. Health Promot Chronic Dis Prev Can. 2020;40(5/6): 139-42. doi:10.24095/hpcdp.40.5/6.02.

10. Wettlaufer A, Vallance K, Chow C, et al. Reducing alcohol-related harms and costs in Manitoba: a policy review. Victoria (BC): Canadian Institute for Substance Use Research, University of Victoria; 2019.

11. Stockwell T, Vallance K, Chow C, et al. Reducing alcohol-related harms and costs in British Columbia: a policy review. Victoria (BC): Canadian Institute for Substance Use Research, University of Victoria; 2019.

12. Myran DT, Chen JT, Bearnot B, Ip M, Giesbrecht N, Rees VW. Alcohol availability across neighborhoods in Ontario following alcohol sales deregulation, 2013-2017. Am J Public Health. 2019;109(6):899-905. doi: 10.2105/AJPH.2019.305014.
13. Van der Maas M, Giesbrecht N, Stoduto G, Orpana H, Geneau R, Mann R. Drinking patterns, alcohol-related harm and views on policies: results from a pilot of the International Alcohol Control Study in Canada. Health Promot Chronic Dis Prev Can. 2020;40(5/6):165-75. doi:10.24095/hpcdp.40.5/6.05.

14. Sherk A, Stockwell T, Chikritzhs T, et al. Alcohol consumption and the physical availability of take-away alcohol: systematic review and meta-analyses of the days and hours of sale and outlet density. J Stud Alcohol Drugs. 2018;79(1):58-67. doi:10.15288/jsad .2018.79.58.

15. Pauly B, Vallance K, Wettlaufer A, et al. Community managed alcohol programs in Canada: overview of key dimensions and implementation. Drug Alcohol Rev. 2018;37(Suppl 1):S1329. doi:10.1111/dar.12681.

16. Subbaraman MS. Substitution and complementarity of alcohol and cannabis: a review of the literature. Subst Use Misuse. 2016;51(11):1399-414. doi:10.3109/10826084.2016.1170145.

17. Eaton DL, Alberg AJ, Goniewicz M, et al.; Committee on the Review of the Health Effects of Electronic Nicotine Delivery Systems. Public health consequences of e-cigarettes. Consensus study report for the US Food and Drug Administration. Washington (DC): US National Academies of Science, Medicine and Engineering; 2018.

18. Callaghan RC, Verdichevski M, Fyfe TM, Gatley JM. Does cannabis use increase the risk of developing cancer in humans? In: Preedy VR, editor. Chapter e9. Handbook of cannabis and related pathologies: biology, pharmacology and treatment. Cambridge (MA): Elsevier Inc - Academic Press; 2016. pp. e80-100.

19. Benoit C, Stengel C, Marcellus L, et al. Providers' constructions of pregnant and early parenting women who use substances. Sociol Health Illn. 2014;36(2):252-63. doi:10.1111 /1467-9566.12106.
20. Benoit C, Magnus S, Phillips R, Marcellus L, Charbonneau S. Complicating the morality discourse: parents' constructions of problematic substance use. Int J Equity Health. 2015;14:72. doi:10.1186/s12939-015 -0206-7.

21. Marcellus L, MacKinnon K, Benoit C, Phillips R, Stengel C. Reenvisioning definitions of success for programs supporting pregnant women with problematic substance use. Qual Health Res. 2015;25:500-12. doi: $10.1177 / 1049732314551058$.

22. Enns A, Orpana H. Autonomy, competence and relatedness and cannabis and alcohol use among youth in Canada: a cross-sectional analysis. Health Promot Chronic Dis Prev Can 2020;40(5/6):201-10. doi:10.24095 /hpcdp.40.5/6.09. 